\title{
Failure to obtain a generation effect during naturalistic learning
}

\author{
MARIE CARROLL \\ Macquarie University, Sydney, N.S.W., Australia \\ and \\ THOMAS O. NELSON \\ University of Washington, Seattle, Washington
}

\begin{abstract}
Many experiments have obtained a generation effect (GE) with various kinds of laboratory items. Six of the present seven experiments failed to find a GE when the responses were answers to general information questions that had been learned by college undergraduates who had either read or generated the answers during learning several days before the retention test. A GE also did not occur when those same answers were used as responses in paired-associate learning and were tested 20 min after learning. The GE appeared only when subjects learned lists of answers in the absence of the question context, followed by recognition testing. Implications of these findings are drawn both for the generality of the GE, especially to the kind of items and naturalistic situations in which learning occurs outside the laboratory, and for the theoretical mechanisms that may underlie the GE in traditional laboratory situations.
\end{abstract}

Although some previous research has not found any difference in eventual recall after learning had occurred through reading as opposed to generating the to-be-acquired responses (e.g., when the responses are nonsense syllables), the typical finding that has been of much current interest and that is called the "generation effect" (GE) is the following: Eventual recall is better after people learn items by generating the responses themselves than after they learn the items by reading the responses as experimenter-provided words. However, as McDaniel, Riegler, and Waddill (1990) recently pointed out, "If the generation effect were restricted to one very particular paradigm, then its potential application (e.g., in educational situations) and illumination of learning and memory processes in general would be of questionable value" (p. 796).

In accord with this point by McDaniel et al. (1990), we describe below our recurring failure to obtain a GE on items and procedures that we had hoped would produce one. On the assumption that the GE would generalize to everyday materials such as general information items (e.g., "What is the capital of Finland?" Answer = Helsinki) that students learn in naturalistic situations, we compared conditions in which people learned those items by generating as opposed to reading the answers. Our initial goal was to determine how the GE affected various aspects of metamemory such as the feeling of knowing and judgments of learning (note-because a precondition for such an investigation was that a GE should occurand this precondition was never fulfilled in any of our cued-recall experiments-we do not mention further any

This research was partially supported by Grant MH32205. Requests for reprints should be addressed to T. O. Nelson, Department of Psychology (NI-25), University of Washington, Seattle, WA 98195. of the metamemory aspects). Because all but one of our attempts failed to produce a generation effect, these findings confirm the possibility of the limited boundary conditions mentioned by McDaniel et al. In our experiments, the GE did not occur for the ordinary factual items and postacquisition retention intervals that are a part of everyday learning in naturalistic situations.

\section{EXPERIMENT 1}

This experiment explored the possibility of a GE for a situation that involved learning unknown general information items followed by a retention test 1 week later. This 1-week delay had already been used in previous literature (Buyer \& Dominowski, 1989) and is the kind of naturalistic situation that we wanted to model (i.e., longterm recall beyond the acquisition session). Moreover, it is needed to insure off-the-ceiling recall of recently learned general information items with this kind of presentation of each answer (Nelson, Gerler, \& Narens, 1984 , p. 291), because off-the-ceiling performance is a necessary condition for the possibility of observing a GE.

\footnotetext{
Method

Subjects and Design. Twenty-eight undergraduates from the University of New England participated in the within-subject blockedlist design that contained a 1-h acquisition session and a 10-min retention session 1 week later. The independent variable was whether a given item was learned by reading the answer or by generating it from clues (described below) that were given by the experimenter.

Materials and Procedure. Items were general information questions selected from the Nelson and Narens (1980) norms, with a difficulty range of .05 to .30 (i.e., the normative percentage of people who correctly recalled the one-word answer to each question ranged between $5 \%$ and $30 \%$ ). The 116 items were divided into two arbitrary sets of 58, designated A and B. Each question was printed
} 
Table 1

Mean Percent-Correct Recall on the Retention Test for Each Condition in Each Experiment

\begin{tabular}{cccc}
\hline & & \multicolumn{2}{c}{ Condition } \\
\cline { 3 - 4 } Experiment & $n$ & Read & Generate \\
\hline 1 & 27 & 37 & 38 \\
2 & 22 & 48 & 49 \\
3 & 18 & 64 & 66 \\
4 & 30 & 63 & 62 \\
5 & & & \\
General information & 47 & 75 & 72 \\
Word pairs & 42 & 34 & 34 \\
6 & 29 & 81 & 84 \\
7 & 24 & 54 & 63 \\
\hline
\end{tabular}

on an index card. There were two versions of each question in Sets $A$ and $B$; one version had only the question on the card, and the other had the question and the correct answer written directly below.

The subjects were run individually and answered questions from both Sets A and B under the read and generate conditions. Assignment of the sets to the two conditions was counterbalanced, as was order of presentation of the two conditions.

During the acquisition session, the experimenter read the question aloud to the subject, who said aloud his or her answer. If the subject's answer was correct, the experimenter read aloud the next question immediately. If the subject's answer was not correct, the experimenter put a card on the table in front of the subject. The question was printed at the top of the card, and either the answer or nothing was printed directly below the question. (The question was not reread aloud in any condition.) In the read condition, the subject had $15 \mathrm{sec}$ to study while looking at the question and answer. In the generate condition, the question appeared alone on the card (and remained visible throughout the generate phase), and the experimenter said aloud the following clues: (1) number of letters in the word, (2) first letter, (3) second letter, (4) third letter, and so forth. After each clue, the subjects were to actively try to generate the word, and guessing was encouraged. The $N+1$ th clue was given only if the subject had not generated the correct answer after the $N$ th clue. If necessary, all letters in the word were eventually spelled out, and the subject had to say aloud the complete word.

Sets were presented in blocks, and the presentation of items during acquisition continued until the subjects had failed to answer 70 questions ( 35 questions from each set). At the end of the acquisition session, the subjects were instructed not to think about or rehearse the answers to the questions during the intervening period.

One week later, they were again asked the 70 questions. Recall was subject-paced, and the subject was encouraged to guess whenever unsure of an answer. Final recall was tested only for items that the subject initially did not recall the answer to and therefore would have to learn in the experiment-either via reading or generating the answer-so that the goal of the experiment was to test memory for general information that people learned in a situation of laboratory control, with the method of that learning being the independent variable. This was designed as a laboratory model of what might happen in an educational situation in which a teacher can choose to teach general information by having the student either read or generate the answers.

\section{Results}

All subjects except one returned for the follow-up test, and therefore recall data for each of the two conditions were obtained from 27 subjects. The mean percentage of items correctly recalled 1 week after acquisition is shown for each condition in the first row of Table 1 . No generation effect appeared; the difference of $1 \%$ in mean re- call for the read versus generate conditions was not significant $(F<1)$.

\section{EXPERIMENT 2}

We had failed to find a GE with general information items when the generation task was to deduce the word from increasingly informative clues, so we decided to use a different manipulation to try to obtain a GE. Experiment 2 contained a generation task that was suggested by G. Mazzoni (personal communication, February 20, 1990 ) and that consisted of generating the anagram solution of the answers to general information questions.

\section{Method}

Subjects. The subjects were 22 introductory psychology students who had not participated in Experiment 1 but who were from the same subject pool. The design was the same as in Experiment 1, except for changes in the items and the method of having the subjects generate answers.

Materials and Procedure. Materials were general information questions selected from the Nelson and Narens (1980) norms at a lesser degree of difficulty (in the range $.30-.50$ ) than in Experiment 1 . Eighty items were divided randomly into Sets $\mathbf{A}$ and $\mathbf{B}$. Following each unsuccessful recall of an item in the generation condition, the anagram of the answer, printed on a separate card, was shown. The degree of scramble of the letters involved rearrangement of the position of almost all of the letters, although very long words did not receive as much scrambling as did short words.

The procedure was the same as in Experiment 1, except that reading or generating unrecalled answers stopped when 20 unrecalled items had been isolated in each set. Pilot work had shown that the new generation manipulation took much longer than in Experiment 1 . When subjects could not solve the anagram in 2 min, the experimenter gave the following clues: first letter, second letter, third letter, and so on. In no case, however, did the experimenter ever have to give the complete solution (i.e., the subjects always generated at least some part of every answer on their own).

\section{Results and Discussion}

The subjects found the anagrams difficult to solve and often required clues. However, the GE again failed to emerge: The second row of Table 1 shows the mean subsequent recall 1 week after acquisition of the 40 items, and again the difference of $1 \%$ in mean recall for the read versus generate conditions was not significant $(F<1)$.

Because a GE had failed to occur in circumstances in which it had previously been found (G. Mazzoni's study, in which a GE effect had occurred that was significant at the .05 level but not at the .01 level; personal communication, February 20, 1990), we considered possible differences between that study and our Experiment 2. First, Mazzoni's study involved less scrambling of letters than did ours, enabling her subjects to solve the anagrams in $7 \mathrm{sec}$. Second, Mazzoni's questions were easier than ours because all of the answers were familiar words. In Experiment 2 , some of the subjects did not recognize the target words even after solving the anagrams, despite our selection of a set that was easier than that in Experiment 1.

It is possible that unfamiliarity of the target word negates any advantage of generating. Therefore, items from Experiment 2 for which the subjects required no let- 
ter clues were isolated for reanalysis on the assumption that rapid solution of the anagram implied some familiarity with the answer. This post hoc selection procedure yielded a set of items that were almost significantly better recalled 1 week later for the generate condition (mean $=57 \%$ ) than for the read condition [mean $=48 \%$; $F(1,26)=3.39, p<.07]$. Perhaps, therefore, item familiarity produces a generation advantage that is obscured when the overall set of items contains both familiar and unfamiliar responses.

\section{EXPERIMENT 3}

We wanted to explore further the possibility that the GE may depend critically on item familiarity and/or fast solution times. Experiment 3 was designed to test the hypothesis that the GE depends on a fast generation of familiar items, as suggested by the post hoc analysis in Experiment 2.

\section{Method}

Subjects. The subjects were 18 undergraduates who had not participated in Experiments 1 or 2 but who were from the same subject pool.

Materials and Procedure. Questions whose answers were not recalled by a majority of the subjects in Experiment 2 were omitted, leaving a pool of 28 of the 40 items in Set B and 29 of the 40 items in Set A. Besides using only familiar items, we reduced the degree of scramble of the letters in the anagram condition to make the generation task somewhat easier than it had been in Experiment 2. The position of only two or three adjacent letters was interchanged, and these letters were never the first letter in the word.

Because there were fewer and easier items than there were in Experiment 2, there was no longer a restriction on the number of unrecalled items that would be tested, therefore all items were tested for every subject.

\section{Results and Discussion}

The manipulation of including only easy anagrams was successful; no subject failed to solve each anagram within $7 \mathrm{sec}$. Nevertheless, the GE did not appear: The $2 \%$ difference between conditions shown in the third row of Table 1 is not significant $(F<1)$. These results indicate that fast solution times and a higher degree of item familiarity are not sufficient to produce a GE with general information questions.

\section{EXPERIMENT 4}

An additional manipulation seemed desirable to try to increase the saliency of the cognitive operations in the generate condition. Experiments 1, 2, and 3 had blocked rather than mixed presentation of items in the read and generate conditions. In Experiment 4, the presentation was mixed: An unrecalled item from Set A was always followed by questions from Set $B$ until an unrecalled answer occurred in Set B. Although blocking of conditions (pure lists) has produced generation effects, other studies have shown that the generation effect occurs only if the read and generated items are intermixed (Slamecka \& Katsaiti, 1987). This is because items read in mixed lists are not remembered as well as items read in pure lists. In Experiment 4 , we hoped that the juxtaposition of read and generated items might sharpen the contrast between them (cf. analogous effects of magnitude of reward on human learning in Nelson, 1976).

\section{Method}

Subjects. A new group of 30 undergraduates from the same subject pool as in Experiments 1-3 participated in Experiment 4.

Materials and Procedure. Materials were the same as in Experiment 3 . In the acquisition phase, when the anagram to an unrecallable generate question in Set $A$ had been solved, questions from Set $B$ would follow until one question failed to be answered correctly, whereupon the unrecalled answer was presented so that the subject could read it, and so on. At test 1 week later, questions were also asked in a mixed fashion.

\section{Results and Discussion}

The fourth row of Table 1 shows that the mean difference in recall on the retention test was only $1 \%$, which was not significant $(F<1)$. Thus, again no GE occurred. Therefore, the GE is not contingent on presentation conditions that emphasize the different encoding operations involved in generating versus reading.

\section{EXPERIMENT 5}

Our repeated failure to obtain a GE with general information questions stands in stark contrast to the many studies that have yielded GEs with materials such as word pairs, word fragments, and single words. Blaxton (1989) reported obtaining a GE with general information questions, but aspects of her method were different from ours. During the test phase of her experiment, a general information question served as the cue for the target (e.g., "What is the name of the lunar landing module?"), but the general information question had not been part of the learning context. We wondered if this difference in the context during learning between Blaxton's and our experiments might account for the different outcomes in regard to the GE.

Perhaps there is something unique about our learning context (the general information question) and the target (the answer) that masks a GE. In particular, it may be that a GE pertains more to material in episodic than in semantic memory. Therefore, Experiment 5 was an attempt to obtain a GE using newly learned word pairs rather than answers to general information questions. In each word pair, the target was the same word as an answer to one of the general information questions in Experiment 4; however, the cue member of the word pair was unrelated to the target. The subject was required to generate the target from an anagram as in Experiment 4 and was required at test to produce that target in response to the same cue that had occurred during learning.

\section{Method}

Subjects. The general information group, which was a replication of Experiment 4, contained 47 new undergraduate subjects, and the word pairs group contained $\mathbf{4 2}$ new undergraduate subjects. 
These subjects were from the same pool as in Experiments 1-4, except that these undergraduates were from Macquarie University.

Materials and Design. For the word pairs group, two sets of word pairs were constructed so that the target member of the pair was the same word as the answer to a general information question in Sets $A$ or $B$ in Experiment 4. The cue member was an unrelated word of high frequency from the Kutera-Francis norms. One version of the sets presented the items in pairs on single cards, with the cue typed above the uppercase target. The second version presented the cue with the scrambled target word below it. The targets were scrambled in the same way as in Experiment 4 . For the general information group, the materials were identical to those in Experiment 4.

The mixed design was between subjects for kind of item (word pairs vs. general information) and within subjects for read or generate at acquisition. As in Experiment 4, the presentation was mixed rather than blocked by condition.

Procedure. During acquisition, the subjects in the general information group were required to learn the response term to unrecalled items by reading or generating, and they were tested 1 week later. The subjects in the word pairs group would of course not know the "answer" to any of the episodic pairs, thus all cue words were presented for testing, followed by one learning episode per pair. Pilot work indicated that recall was too close to the floor (approximately $15 \%$ correct) when the word pair group was tested 1 week later. Therefore, we decided to test the word pair group following a 10-min distractor task presented immediately after learning, in order to raise the overall recall rate to a level more similar to that for the general information conditions.

\section{Results and Discussion}

As indicated in the fifth row of Table 1, a GE did not appear in the general information group; the mean difference of $3 \%$ was not significant $(F<1)$. There was also no GE in the word pair group; the sixth row of Table 1 shows that mean recall was identical after learning through generation as opposed to reading.

Experiment 5 failed to find a GE using newly learned word pairs, in which the target was the answer to a general knowledge question. Therefore, the failure to find a GE is not due to the difference between semantic (general knowledge questions) versus episodic (word pairs) tasks.

\section{EXPERIMENT 6}

A further possibility for the failure to obtain a $G E$ is that the cued-recall test that we used in the preceding experiments is not sufficiently sensitive to produce a GE. For this reason, we attempted an alternative method of testing, that of uncued recognition of single items. In Experiment 6 , then, the questions and answers were presented for study as in all other experiments, but instead of a cued-recall test 1 week later, the test involved freechoice recognition of a list of targets and distractors.

\section{Method}

Subjects. A new group of 29 undergraduates participated.

Materials and Procedure. Materials and the procedure at acquisition were identical to those used in Experiment 4. At acquisition, when the anagram to an unrecallable generate question in Set A had been solved, questions from Set B would follow in a mixedpresentation fashion.

However, the test session, conducted 1 week later, involved a free-choice recognition test. All target words from the previous ac- quisition session, whether correctly recalled or not, were intermixed with an equal number of distractors and were presented on a sheet of paper. The subjects were instructed to circle all words that they recognized as answers to the questions in the earlier session. Unlimited time was given to complete the task. A set of distractors was chosen that matched each target in meaning (e.g., targetHOCKEY; distractor-SOCCER; AMAZON-NILE; ARGENTINA- PERU).

\section{Results and Discussion}

A GE failed to appear, even with a recognition test. The seventh row in Table 1 shows that there was a high level of recognition of targets for items that had previously been unrecalled but that the difference was not significantly different for the read and generate conditions $[t(28)=.81, p>.05]$.

\section{EXPERIMENT 7}

As a consequence of this further failure, we tested an additional 24 subjects under identical conditions, with one exception: At study, no question occurred as a context for the target. The subjects received the same recognition test 1 week later but either read or generated the targets alone at study. Targets from all questions were, of course, included, since there were no "unrecallable" answers. What we obtained here (see the eighth row in Table 1) showed that this particular set of targets is not some unique set of words that can never show a GE. The generated words (mean proportion correct $=.63$ ) were recognized significantly better than were the read (mean proportion correct $=.54$ ) words 1 week later $[t(23)=$ $2.65, p=.01]$. We have thus identified a boundary condition for the GE: It occurs for single words that are studied out of context and tested by recognition, but it does not occur when those same words are studied in the context of general knowledge questions to which those words are the answers, either when tested via cued recall or by recognition.

\section{GENERAL DISCUSSION}

All rows in Table 1, except the last, show a definite lack of a GE for the items and procedures that we investigated. Next, we consider several theoretical mechanisms for the GE that have been proposed in the current literature. Although our results may not be critical for any given theoretical mechanism, they do have implications for the feasibility of those mechanisms as general explanations of the GE.

\section{Possibility 1: Unfamiliarity of the Items}

One possible explanation for our results is the potential unfamiliarity of some of the target items. Slamecka and Fevreiski (1983) suggested that generation will have a memorial advantage only when the generated unit already has a representation in the subjective lexicon-that is, when it is already familiar. However, Gardiner and Hampton (1985) showed that prior familiarity is not a necessary condition for the GE. Rather, it is important for the item to be represented in semantic memory as an in- 
tegrated functional unit (i.e., perceived and encoded as a familiar concept). The subjects' successful solutions on our anagram task demonstrate that each of the targets for our items is indeed perceived as a unit in memory.

Another obvious candidate is our use of general information questions. However, as mentioned above, Blaxton (1989) obtained a GE using these stimuli, but her design entailed changing the background context (for the target) between study and test, whereas in our experiments, the context remained the same (as it presumably would in vivo when people are studying items for upcoming classroom tests). In the limited set of studies available in the literature, the only reported failure to find a GE using material similar to ours was in an experiment by Nairne, Pusen, and Widner (1985). Subjects were taught definitions to experimenter-created items even though they understood each item's assigned semantic properties. However, our general information questions were not as artificial as were their stimuli, and lack of familiarity is not an obvious explanation of our results.

\section{Possibility 2: Delay Between Study and Test}

Buyer and Dominowski (1989), using common-knowledge phrases in response to numbers (e.g., 26-letters of the alphabet) found a GE after a 1-week retention interval. Thus, our failure to find a GE does not seem to be due to a 1-week retention interval.

\section{Possibility 3: Effort}

An early explanation for the GE suggested by Tyler, Hertel, McCallum, and Ellis (1979) was the increased effort in generating, which may produce the memorial advantage. In support of this view, subsequent research (see Gardiner, Smith, Richardson, Burrows, \& Williams, 1985; also see Buyer \& Dominowski, 1989) using word completion as the generating task-similar to the letterclues condition in our Experiment 1-showed that increases in retention occurred with increased numbers of letters required for completion of a word. They argued that increases in retention are the result of increases in item difficulty, which is in accord with the effort hypothesis. However, our experiments showed that there was no GE when the generate task was to deduce the word from letter clues. Indeed, the only hint of a GE in our cued-recall experiments occurred in Experiment 2 when those items that had been generated most easily were analyzed apart from other generated items. This suggests that too little effort in the generation condition is not the reason for our lack of a GE. Moreover, when our generation task was changed to an anagram solution task, neither easy (Experiments 3, 4, 5, and 6) nor difficult (Experiment 2) anagrams yielded a GE.

\section{Possibility 4: Relationship Between the Cue and the Target}

There is conflicting evidence about the importance of the relationship between cue and target for the occurrence of a GE. The results of some experiments suggest that the cue and target must be related for the GE to appear. This was supported by Hirshman and Bjork (1988), who found recall enhanced for generated material when the response bore an orthographic, acoustic, or semantic relation to a stimulus word. Nairne, Pusen, and Widner (1985) found no GE when nonwords were generated, and they argued that a necessary condition for the GE is how related the generated item is to other potential retrieval cues in the memory system. Subsequently, Nairne and Widner (1988) found that low-frequency words did produce significant retention advantages when generated, but only when the items were rated as highly familiar. Our Experiments 4 and 5 included only easy general information questions, and it is likely that all of the subjects were familiar with the correct response, once learned.

Several investigators (Nairne \& Widner, 1987; Rabinowitz \& Craik, 1986) have emphasized the importance of matching the context of what is generated during study with the context of what is tested during retention. In our experiments, solving an anagram at study was never matched by the same operations at test. Moreover, although Blaxton (1989) kept the meaning of the target relatively constant from study to test for most items, she obtained a GE even when some of the items had a change in the meaning of the target word generated at study (e.g., eagle as a type of bird) to a different meaning at test (eagle as a lunar landing module) and most important for contrasting her procedure with ours, she used general information questions as the stimuli only during the retention test and not during study.

A related possibility is that the generation task has to focus more on the meaning (as opposed to the label) of the target; for example, perhaps a generation effect would emerge with our items and procedure if subjects were given semantic cues about the target (we are grateful to Teresa Blaxton for suggesting this possibility; personal communication, June 9,1992 ). However, we should mention that previous research showed that the relationship between the cue and target does not necessarily have to be semantic for a GE to emerge; for instance, Gardiner and Rowley (1984) obtained a GE when subjects remembered answers to multiplication sums, and Johns and Swanson (1988) obtained a GE regardless of the lexical status of the cue and target.

\section{Possibility 5: Generating Achieves Its Advantage Because Reading Yields Less Semantic \\ Processing of the Items}

Perhaps the most promising explanation for our results is related to Jacoby's (1978) idea that the GE occurs because generating (compared with reading) produces greater semantic processing of the meaning of the to-beacquired items. General information questions may be more interesting than most laboratory items, so that even those subjects in the read-the-experimenter's-answers condition do a thorough semantic processing of the items. It would be difficult to use this explanation to account for the lack of a GE for unrelated pairs in Experiment 5, un- 
less the subjects in the word pairs group also tried to construct a semantically meaningful relation, which may be the case for the proper nouns that we used. Our use of material that was interesting and/or nontrivial may have led to some kind of disinhibition for the people in our read condition. Although this may provide a theoretical explanation of our findings, if correct, it would have severe implications for the generalizability of the GE to naturalistic learning situations.

\section{Conclusion}

There does not seem to be any theoretical explanation for our repeated failure to obtain a GE that does not also have unfortunate implications for extensions to educational situations (see the quotation from McDaniel et al., 1990, at the beginning of this article). In Experiments 1-6, eventual retention was no better after generating than after reading. Moreover, relative to the items from most previous experiments, our items are most similar to what learners typically acquire in naturalistic situations.

Although the GE may be a reliable phenomenon in laboratory situations, a major concern of experimental psychologists is that a given laboratory phenomenon may occur only in restricted situations in the laboratory. If the $\mathrm{GE}$ is due more to some kind of lack of attention and/or lack of semantic processing in the reading control condition-rather than to some kind of facilitation of memory in the generation condition-it loses much interest as a general principle that can help normal learners who already attend to what they are trying to learn and who are seeking better ways to improve their intentional learning.

Ours is the first attempt of which we are aware to generalize the GE to the kind of items that occur in naturalistic learning situations. Our findings suggest a serious lack of generality for the GE because when the items are general information questions, eventual recall is no better after generating the answers than after a traditional reading of the answers.

\section{REFERENCES}

Blaxton, T. A. (1989). Investigating dissociations among memory measures: Support for a transfer-appropriate processing framework. Journal of Experimental Psychology: Learning, Memory, \& Cognition, 15, 657-668.

BUyer, L. S., \& Dominowski, R. L. (1989). Retention of solutions: It is better to give than to receive. American Journal of Psychology, 102, 353-363

Gardiner, J. M., \& HAMPTon, J. A. (1985). Semantic memory and the generation effect: Some tests of the lexical activation hypothesis. Journal of Experimental Psychology: Learning, Memory, \& Cognition, 11, 732-741.

Gardiner, J. M. \& Rowley, J. M. C. (1984). A generation effect with numbers rather than words. Memory \& Cognition, 12, 443-445.

Gardiner, J. M., Smith, H. E. C., Richardoson, C. J., Burrows, M. V., \& Williams, S. D. (1985). The generation effect: Continuity between generating and reading. American Journal of Psychology, 98, 373-378

Hirshman, E., \& Bjork, R. A. (1988). The generation effect: Support for a two-factor theory. Journal of Experimental Psychology: Learning, Memory, \& Cognition, 14, 484-494.

JACOBY, L. (1978). On interpreting the effects of repetition: Solving a problem versus remembering a solution. Journal of Verbal Learning \& Verbal Behavior, 17, 649-667.

JoHNS, E. E., \& SWANSON, L. G. (1988). The generation effect with nonwords. Journal of Experimental Psychology: Learning, Memory, \& Cognition, 14, 180-190.

McDaniel, M. A., Riegler, G. L., \& WaddiLl, P. J. (1990). Generation effects in free recall: Further support for a three-factor theory. Journal of Experimental Psychology: Learning, Memory, \& Cognition, 16, 789-798.

Nairne, J. S., Pusen, C., \& Widner, R. L., JR. (1985). Representation in the mental lexicon: Implications for theories of the generation effect. Memory \& Cognition, 13, 183-191.

NAIRNE, J. S., \& WIDNER, R. L. (1987). Generation effects with nonwords: The role of test appropriateness. Jourmal of Experimental Psychology: Learning, Memory, \& Cognition, 13, 164-171

NaIRNE, J. S., \& WidNER, R. L. (1988). Familiarity and lexicality as determinants of the generation effect. Journal of Experimental Psychology: Learning, Memory, \& Cognition, 14, 694-699

NELSON, T. O. (1976). Reinforcement and human memory. In W. K. Estes (Ed.), Handbook of learning and cognitive processes (Vol. 3, pp. 207-246). Hillsdale, NJ: Erlbaum.

Nelson, T. O., Gerler, D., \& Narens, L. (1984). Accuracy of feelingof-knowing judgments for predicting perceptual identification and relearning. Joumal of Experimental Psychology: General, 113, 282-300.

Nelson, T. O., \& Narens, L. (1980). Norms of 300 generalinformation questions: Accuracy of recall, latency of recall, and feeling-of-knowing ratings. Journal of Verbal Learning \& Verbal Behavior, 19, 338-368.

Rabinowitz, J. C., \& Craik, F. I. M. (1986). Specific enhancement effects associated with word generation. Journal of Memory \& Language, 25, 226-237.

Slamecka, N. J., \& Fevreiski, J. (1983). The generation effect when generation fails. Journal of Verbal Learning \& Verbal Behavior, 22, 153-163.

Slamecka, N. J., \& Katsaiti, L. T. (1987). The generation effect as an artifact of selective displaced rehearsal. Joumal of Memory \& Language, 26, 589-607.

Tyler, S. W., Hertel, P. T., McCallum, M. C., \& Ellis, H. C. (1979). Cognitive effort and memory. Joumal of Experimental Psychology: Learning, Memory, \& Cognition, 5, 607-617.

(Manuscript received September 3, 1991; revision accepted for publication September 16, 1992.) 